\title{
Investigating the knowledge, perception, and practice of healthcare practitioners toward rational use of compounded medications and its contribution
} to antimicrobial resistance: a cross-sectional study

Desta Assefa*, Getahun Paulos, Dereje Kebebe, Sintayehu Alemu, Wondu Reta, Temesgen Mulugeta and Fanta Gashe

\begin{abstract}
Background: Pharmaceutical compounding ensures access of individuals with specific requirements to individualized therapy. However, there is an inconsistency of compounded medication quality. Therefore, advancing the rational use of compounded medication is essential for patient safety and medication effectiveness.

Objective: The presented study was aimed to investigate the healthcare practitioners'knowledge, perception, and practice of extemporaneous compounding and its contribution to the prevalence of antimicrobial resistance.

Method: A descriptive cross-sectional survey using a structured questionnaire was conducted. The study participants were 300 healthcare practitioners working in Jimma University Medical Center, hospital pharmacies, and community pharmacies in Jimma and Mettu Town, Southwest Ethiopia.

Results: Most respondents were pharmacists (62.7\%) and first-degree holders (48.3\%). The majority of them had experience in administering (57.7\%), preparing (38\%), prescribing (21\%), and repackaging and labeling (14\%) compounded medications. Commonly they request compounded medications when prepackaged products (77.7\%) and needed dosage regimens (72.3\%) were not available in the market. However, most of them believed that compounded medications might lack quality (49\%) and had poor patient compliance (40.7\%). Moreover, they fear that inappropriate preparation processes (75\%) and under-dose administration (59\%) of compounded medication might contribute to the development and prevalence of antimicrobial resistance.
\end{abstract}

Conclusion: Most healthcare practitioners practice rational use of compounded medications and strongly agree that inappropriate compounding of antimicrobials contributes to antimicrobial resistance development.

Keywords: Antimicrobial resistance, Compounding, Healthcare practitioners, Rational use

${ }^{*}$ Correspondence: desta4best@gmail.com

School of Pharmacy, Jimma University, Oromia, Ethiopia

\section{Introduction}

Ensuring medication availability in healthcare facilities (HCFs) is crucial to providing good quality healthcare and reducing avoidable patient readmissions. In addition 
to manufacturing in the licensed pharmaceutical industries, extemporaneous compounding is the essential source of medication availability, especially for personalized healthcare [1-4].

Since the mid-1980s, the number of patients seeking personalized healthcare has been increasing. It is due to one size medicine (strength and dosage form) does not fit all. Therefore prescribing, dispensing, and administering extemporaneously compounded medication is a good solution. Extemporaneous compounding is the art and science of combining, mixing, repackaging, and labeling a drug product. Historically, it has been a crucial part of the pharmaceutical process. It has long been a service provided by licensed pharmacists since the origin of pharmacy. Today, with the technological advancements in the pharmaceutical field and increased need, the compounding pharmacy industry is experiencing a resurgence and becoming more relevant than ever [3,5-11].

The medicine compounders compound according to a licensed practitioner's prescription, medication order, or initiative based on the practitioner-patient-pharmacist relationship in the course of professional practice $[1,12]$. It includes removal of allergenic ingredients, dosage form change, especially for easy swallow, altering strength, routes of the delivery, unpleasant flavor of available drug product, and allowing access to discontinued medications [5-7, 9, 12-14]. Therefore, compounding allows specific patients such as pediatric and geriatric patients, patients requiring dermatologic disease treatment and pain management, and others with more options $[1,8]$.

The compounded medications should be developed to meet unique medical needs and ensure patient safety. Therefore, compounding prescriptions should be prescribed by a licensed practitioner, compounded and dispensed by the licensed pharmacist, administered and used correctly. Compounding pharmacies must also comply with good compounding practice regulations for compounding within hospitals, community pharmacies, home infusion pharmacies, and, more recently, outsourced compounding facilities $[1,8,9]$.

The regulatory scrutiny of pharmaceutical compounding practices is significantly less rigorous than that required for FDA-approved medications (commercially available medications). It is exempted from regular inspections, quality control testing, and rejection of material not meeting specifications. Furthermore, they are not clinically assessed for safety or efficacy before marketing. Moreover, there is no standard labeling or prescribing information with instructions for the safe use of compounded products. They are the potential causes for poor quality medication preparation (contamination, medication with too little or too much active ingredient, or failure to meet specifications). Finally, they may pose potentially fatal health risks for the users $[5,6,12$, 15-19]. As evidence, FDA reported the prevalence and potential risks of poor quality compounded medications many times. These defects lead to serious patient illnesses, adverse events, and deaths linked to poor quality compounded drugs [16, 20, 21].

Poor quality of compounded medication causes administration of a low dose of active ingredients. Administration of lower-dose antimicrobials can cause microbes, including bacteria, fungi, parasites, and viruses, to adapt and become less susceptible to medical treatment. Therefore, it can play a role in the emergence and spread of antimicrobial resistance (AMR), which causes a profound threat to human health. Moreover, irrational prescribing, packaging and labeling, administering, and using compounded medicine can contribute to AMR prevalence $[1,22-24]$. Thus, the rational use of compounded antimicrobials can be the critical action to prevent the spread of antimicrobial-resistant infections. It mostly depends on healthcare practitioners' (HCPs') knowledge, perceptions, and practices. The presented study was aimed to investigate their knowledge, perception, and practice of extemporaneous compounding and its contribution to the prevalence of AMR.

\section{Methods}

\section{Study design and settings}

A descriptive cross-sectional study was conducted from March 15 to May 25, 2021, among the HCPs working in selected HCFs. The HCFs were Jimma University Medical Center, hospital pharmacies, and community pharmacies located in Jimma Town and Mettu Town. These towns are located at $346 \mathrm{~km}$ (Jimma Town) and $600 \mathrm{~km}$ (Mettu Town) from Addis Ababa, the Capital City of Ethiopia in the Southwest. The study settings have been giving healthcare services to a large population. They were selected through convenience sampling.

Jimma University Medical Center is the only teaching and referral hospital in the southwestern part of Ethiopia. With 800-bed capacity and hospital pharmacies, it provides healthcare services for inpatient and outpatient attendants coming to the hospital from the catchment population. Moreover, community pharmacies in study settings are giving pharmaceutical care. It includes compounding pharmaceutical products and dispensing.

\section{Study participants and sampling method}

The study participants were HCPs such as physicians, pharmacists, nurses, medical laboratory technologists, and midwives. They were chosen since the study focused on one of the most important elements of healthcare. So, they should be aware of the need for compounded medications and their safety. Additionally, they should 
be encouraged to report potential risks linked to poor quality compounded drugs and effectively protect the patients.

The voluntary study participants were selected and sampled using the convenience sampling technique. Accordingly, 243 HCPs from Jimma Town and 57 (19\%) pharmacists from Mettu Town were involved in the study. Due to pharmacy professionals being formally licensed and experienced professionals for compounding both sterile and non-sterile medications $[1,25]$, most of the participants $(188$ (62.7\%) were pharmacists.

\section{Data collection}

Data was collected using a self-administered structured questionnaire. It was constructed using the English language after an intensive review of published relevant literature [24, 26, 27]. The developed questionnaire had three sections containing closed-ended questions. The first section asked about the respondents' demographic characteristics. The second section described their experience of practicing rational use of extemporaneously compounded antimicrobial medications, reasons for performing antimicrobial compounding, and not practicing their rational use. The third section asked about the perception and knowledge of the respondents on the contributions of extemporaneously compounded antimicrobials to the development of antimicrobial resistance.

A pilot study was conducted using the first draft of the survey instrument. Randomly selected four respondents from each HCF have filled the questionnaire and commented on the clarity of each question. Their comments and responses were evaluated and used to guide revisions. Finally, minor editions were performed to avoid the ambiguity of the questionnaire. Data obtained from the pilot study was not included in the final analysis.

The principal and co-investigators provided training for data collectors (pharmacists and nurses). Before data collection, potential study participants were verbally provided all pertinent information about the study. After allowing them ample opportunity to ask for uncertainty, data collectors provided the data collection sheet. Following sufficient time for reading, the study participants were asked for their voluntarism and requested to agree to verbal informed consent before filling the questionnaire. The data collector supplies the questionnaire for the voluntary participant and collects it after fill. The collected data were checked for completeness and de-identified to ensure confidentiality.

\section{Data analysis}

After collecting the filled questionnaire, data were entered and descriptively analyzed using Statistical Package for the Social Sciences (SPSS), version 21.0.
Descriptive analysis, such as frequency distribution and percentage, was used to analyze categorical data.

Inclusion criteria: Voluntary HCPs on duty during the study period were contacted and included in the study.

Exclusion criteria: Involuntary HCPs were excluded from the study.

\section{Operational definition}

Extemporaneous compounding: Small scale preparation, altering, mixing, repackaging, and labeling of medication with prescriber's instructions [1].

Rational use of compounded medication: Compounded medication accomplishing key elements of rational drug use such as effective, acceptable quality, acceptable safety, accurate prescribing (drug, dose, interval, and duration), appropriate and timely administration, affordable and dispensed correctly for the right patient [28].

\section{Results}

The present study was conducted using 300 study participants who were voluntarily consented to participate in the study. They were working at different units at Jimma University Medical Center, such as hospital pharmacies and wards (148), Jimma Town community pharmacies (95), and Mettu Town community pharmacies (57). The respondents had worked as healthcare professionals for a mean of $5.78 \pm 4.6$ years (range $1-32$ years) (Table 1 ).

The present study indicated that all the HCPs mentioned different reasons for requesting and performing compounding medication and their route of administration (Table 2).

Multiple response analysis indicated that 262 (87.33\%) of the study participants had participated in at least one process of rational use of extemporaneously compounded medications (Fig. 1). They include administering 173 (44.1\%\%), preparing 114 (29.1\%), prescribing 63 $(16.1 \%)$, and repackaging and labeling of compounded medications 42 (10.7\%), respectively. $91 \%$ of trained respondents on extemporaneous compounding of medication had practiced compounding services, especially administering (44, $p=0.60$ and preparing (30) $(p=0.53)$. Most first-degree and specialty (master degree) holders had practiced more on prescribing (47), preparation (75), and repackaging and labeling (27) of compounded medications. However, most diploma holders (91) had practiced administration of compounded medications.

The study respondents practiced rational use of extemporaneously compounded antimicrobials at different frequencies. Most of them practiced like monthly (occasionally) 108 (36\%), almost every day 93 (31\%), not at all $77(25.7 \%)$ and always 22 (7.3\%), respectively. Most of the 
Table 1 Demographic characteristics of healthcare practitioners in the healthcare facilities

\begin{tabular}{|c|c|c|c|c|c|}
\hline \multicolumn{2}{|l|}{ Characteristics } & \multirow{2}{*}{$\begin{array}{l}\text { No. }=\mathbf{3 0 0}, \mathbf{1 0 0 \%} \\
176(58.7)\end{array}$} & \multicolumn{2}{|l|}{ Characteristics } & No. $=300,100 \%$ \\
\hline \multirow[t]{2}{*}{ Sex } & Male & & Respondent profession & Physician & $28(9.3)$ \\
\hline & Female & $124(41.3)$ & & Medical laboratory & $24(8)$ \\
\hline \multirow[t]{4}{*}{ Age (years) } & 20-30 & $175(58.3)$ & & Nursing & $54(18)$ \\
\hline & $30-35$ & $66(22)$ & & Pharmacy & $188(62.7)$ \\
\hline & $35-40$ & $23(7.7)$ & & Midwives & $6(2)$ \\
\hline & $>40$ & $36(12)$ & & & \\
\hline \multirow[t]{4}{*}{ Educational level } & Diploma & $142(47.3)$ & Work experience (years) & $<5$ & $151(50.3)$ \\
\hline & First degree & $145(48.3)$ & & $5-10$ & $92(30.7)$ \\
\hline & \multirow{2}{*}{$\begin{array}{l}\text { Specialty (Master } \\
\text { degree) }\end{array}$} & $13(4.4)$ & & $>10$ & $57(19)$ \\
\hline & & & \multicolumn{2}{|c|}{ Took training on compounding medications } & $73(24.3 \%)$ \\
\hline
\end{tabular}

Table 2 Reasons for performing extemporaneous compounding of medications and route of administration

\begin{tabular}{lc}
\hline Reasons for performing extemporaneous compounding of medicine & No. $=\mathbf{3 0 0 , \mathbf { 1 0 0 \% }}$ \\
\hline Unavailability of prepackaged products in the market & $233(77.7)$ \\
Unavailability of needed dosage regimen (dose, form) (individualized therapy) & $217(72.3)$ \\
The product was discontinued by the manufacturer & $81(27)$ \\
To combine two or more active ingredients into one product & $128(42.7)$ \\
To improve adherence to medications (flavor, taste) & $68(22.7)$ \\
Allergy to the excipient(s) in the commercially available product & $87(29)$ \\
Compounded medications are less expensive & $90(30)$ \\
Ministry of Health encourages compounding & $65(21.7)$ \\
Improving the stability of the desired product & $61(20.3)$ \\
Route of administration for compounded medication & $214(71.3)$ \\
Oral & $148(49.3)$ \\
Ophthalmic & $121(40.3)$ \\
Topical (Skin) & $89(29.7)$ \\
Parenteral & $82(27.3)$ \\
Otic & $44(14.7)$ \\
Rectal & $32(10.7)$ \\
Nasal & $24(8)$ \\
Inhalation & $44(14.7)$ \\
Rectal & $5(1.7)$ \\
\hline
\end{tabular}

time, the respondents didn't prescribe, dispense or prepare antimicrobial medicine extemporaneously due to different challenges (Table 3).

The present study showed the perception of HCPs on the development of AMR due to antimicrobial compounding. Moreover, most of them have knowledge concerning the compounding factors contributing to AMR development (Table 3).

\section{Discussion}

Pharmaceutical compounding has long been an essential component of health care. So, rational use of compounded medications is a fundamental activity within HCFs (9). The main findings of the present investigation showed that most of the HCPs participated in the preparation and administration of the compounded medications (Fig. 1). Pharmacy professionals were the most 


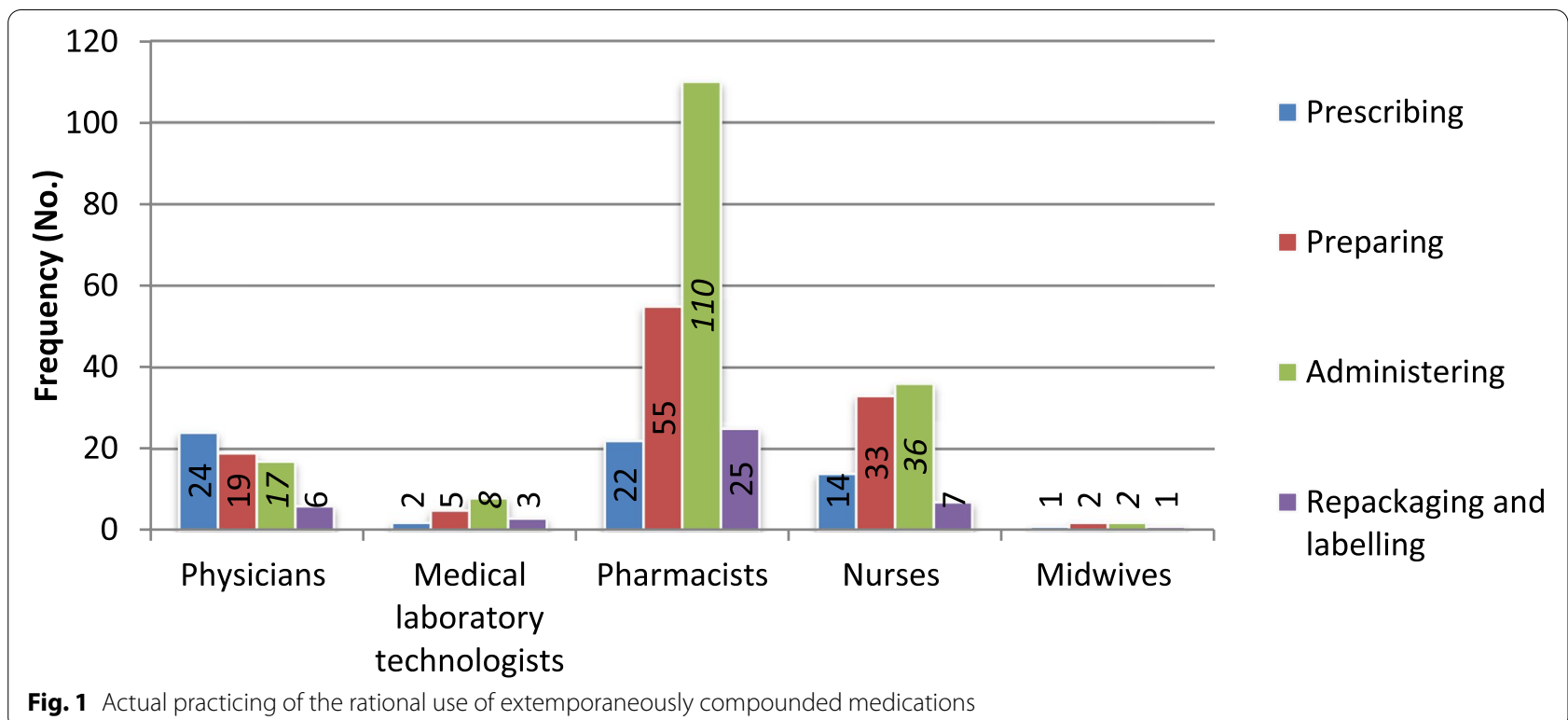

Table 3 Challenges to practice rational use of extemporaneously compounded antimicrobial medicine

\begin{tabular}{lll}
\hline Challenges & No. $=\mathbf{3 0 0 , \mathbf { 1 0 0 } \%}$ \\
\hline Lack of trust in the quality of the compounded formulations & $147(49)$ & $122(40.7)$ \\
Belief that compounded formulations have poor patient compliance & $49(16.3)$ \\
Compounded prescriptions are inconvenient economically & $33(11)$ \\
Belief that the ministry of health does not allow compounded formulations & skilled personnel & $80(26.7)$ \\
not adequate & manufacturing environment & $50(16.7)$ \\
& no ingredients & $67(22.3)$ \\
Fear of inappropriate use (overuse or underuse) & & $73(24.3)$ \\
Fear of AMR development & & $3(1)$ \\
Other (specify) & & \\
\hline
\end{tabular}

common practitioners. It might be due to pharmacists being formally trained in the art and science of compounding medications in their undergraduate courses and licensed to compound medicine relative to other medical communities [1, 23]. Moreover, a limited number of respondents took training on the compounding of medications.

An earlier study has linked the prevalence of compounding services to requests for individualized therapy $[29,30]$. Similarly, the present study showed that the most common reasons for requesting and providing compounding services are the unavailability of FDAapproved drugs in the prepackaged form in the market and the needed dosage regimen for individualized therapy (Table 2). Moreover, the availability of requested dosage regimens for individualized therapy contributes to users' adherence and therapeutic efficacy.
Various studies indicated the high demand for pharmaceutical compounding services at different levels of HCFs. However, the service is not prevalent due to several challenges [10, 31]. Accordingly, the present study reported HCPs' lack of trust in the quality of the compounded formulations and fear of poor patient compliance as common challenges (Table 3). This finding agreed with a study conducted by Abdel Naser Zaid et al. (2010) [31]. It can be due to the quality of compounded products is not regulated by the Ethiopian FDA. Moreover, patients who suffer from the side effects of the compounded medication may bring negligence and malpractice claims against HCPs [16]. As a result, HCPs should be careful during providing rational use of compounded products.

Previous studies reported a lack of prescriptions for requesting compounding services as one of the key 
Table 4 Perception and knowledge of healthcare practitioners on the contributions of antimicrobial compounding to antimicrobial resistance

\begin{tabular}{|c|c|c|c|c|c|c|c|}
\hline \multirow{2}{*}{\multicolumn{2}{|c|}{ Statements for perception evaluation }} & \multicolumn{6}{|l|}{ №. $=300,100 \%$} \\
\hline & & strongly agree & Agree & Neutral & Disagree & Strongly disagree & Don't know \\
\hline \multicolumn{2}{|c|}{$\begin{array}{l}\text { More cautious use of antimicrobials in extemporaneous formu- } \\
\text { lations would decrease AMR }\end{array}$} & $93(31)$ & $95(31.7)$ & $45(15)$ & $6(2)$ & $21(7)$ & $40(13.3)$ \\
\hline \multicolumn{2}{|c|}{$\begin{array}{l}\text { For extemporaneous compounding, using broad-spectrum } \\
\text { antimicrobials can be used in place of narrow-spectrum antimi- } \\
\text { crobials to reduce resistance }\end{array}$} & $37(12.3)$ & $82(27.3)$ & $101(33.7)$ & $46(15.3)$ & $12(4)$ & $22(7.3)$ \\
\hline \multicolumn{2}{|c|}{$\begin{array}{l}\text { Inappropriate or substandard extemporaneous compounding } \\
\text { of antimicrobials contributes to AMR development }\end{array}$} & $112(37.3)$ & $46(15.3)$ & $49(16.3)$ & $11(3.7)$ & $20(6.7)$ & $62(20.7)$ \\
\hline \multicolumn{2}{|c|}{ Statements for knowledge evaluation } & \multicolumn{6}{|l|}{$\mathrm{No}=300,100 \%$} \\
\hline \multirow{7}{*}{$\begin{array}{l}\text { Factors contribution for AMR } \\
\text { development }\end{array}$} & Under dose administration & $177(59)$ & & & & & \\
\hline & Contamination & $86(28.7)$ & & & & & \\
\hline & $\begin{array}{l}\text { Inappropriate preparation } \\
\text { process due to lack of standard } \\
\text { operating procedures }\end{array}$ & $225(75)$ & & & & & \\
\hline & $\begin{array}{l}\text { Ineffectiveness nature of com- } \\
\text { pounded preparation }\end{array}$ & $47(15.7)$ & & & & & \\
\hline & $\begin{array}{l}\text { Incompatibility between drug } \\
\text { and excipient(s) }\end{array}$ & $46(15.3)$ & & & & & \\
\hline & Using after beyond use date & $44(14.7)$ & & & & & \\
\hline & Other (specify) & $2(0.7)$ & & & & & \\
\hline
\end{tabular}

reasons for not providing compounding services $(27,32)$. However, the present study did not. It might be due to the prescribers' lack of confidence in the quality and effectiveness of the compounded medication.

Extemporaneously compounded medications are not undergoing regular inspections, quality control testing, and rejection of material not meeting specifications. However, their quality should be regulated to ensure safety [12]. The present study showed that most respondents strongly believed that substandard extemporaneous preparation of antimicrobials contributes to AMR development. They mentioned inappropriate preparation processes, under-dose administration, and contamination during compounding as the factors causing AMR development (Table 4). The responses agreed to the fact that a low dose of active ingredients in poor quality of compounded medications causes microbes to adapt and become less susceptible [1, 19-21].

\section{Conclusions}

The present study indicated that most healthcare practitioners provide healthcare services using compounded medications. They apply rational use principles mainly for administering and preparing compounded medications. Unavailability of prepackaged products in the market and unavailability of needed dosage regimens were the most common reasons mentioned for practicing rational use of compounded medication. However, lack of trust in the quality of the compounded medication and poor patient compliance was the most listed challenges. The majority of them revealed that compounding caused inappropriate preparation processes, and under-dose medication administration can lead to the development and prevalence of antimicrobial resistance.

\section{Recommendation}

Due to individualized therapy is being in high demand, compounding services are becoming critical. Thus, healthcare practitioners should take training on extemporaneous compounding. Moreover, compounding services should be incorporated into professional development and continuing education programs, and the quality of compounded medication should be regulated.

\section{Limitation of the study}

The present study did not statistically analyze the degree of correlation between challenges and reasons of compounding to compounding practices. It can be a basic study point for future researchers.

\section{Abbreviations}

AMR: Antimicrobial resistance; HCP: Healthcare practitioner; HCF: Healthcare facility.

\section{Acknowledgements}

The authors wanted to acknowledge study participants for voluntarily participating and Jimma University for funding us. 


\begin{abstract}
Authors' contributions
All the authors made substantial contributions to conception and design, acquisition of data, or analysis and interpretation of data; took part in drafting the article or revising it critically for important intellectual content; agreed to submit to the current journal; gave final approval of the version to be published; and agree to be accountable for all aspects of the work. The author(s) read and approved the final manuscript.
\end{abstract}

\section{Funding}

Jimma University has funded the authors to conduct the study.

\section{Availability of data and materials}

Data and supplementary materials are readily available from the corresponding author on reasonable request.

\section{Declarations}

\section{Ethics approval and consent to participate}

Written ethical permission to conduct the study by designed research protocol was requested from the Institutional Review Board of Institute of Health, Jimma University, Ethiopia. Additionally, the investigators asked the review board to permit verbal consent to save data collection time. Then Institutional Review Board of the Institute of Health, Jimma University, reviewed the request, granted ethical approval, and confirmed waives for a requirement of written consent (RF; IHRPG1/1029/2020) on January 8, 2021. So, before data collection, the study participants were asked for verbal informed consent. All methods were carried out following relevant guidelines and regulations [32].

\section{Consent for publication}

Not applicable.

\section{Competing interests}

The authors declare that they have no competing interests.

Received: 24 August 2021 Accepted: 15 February 2022

Published online: 23 February 2022

\section{References}

1. Compounding pharmacy of America. What-is-compounding? https:// compoundingrxusa.com/what-is-compounding/. Accessed July, 2021

2. Siamidi A, Pippa N, Demetzos C. Pharmaceutical compounding: Recent advances, lessons learned and future perspectives. Glob Drugs Therap. 2017;2(2):1-3.

3. Drazen JM, Curfman G, Baden LR, Morrissey S. Compounding Errors. N Engl J Med. 2012;367(25):2436-7.

4. Masupye EM, Suleman F, Govender T. Investigating extemporaneous compounding practices in the Polokwane tertiary hospital pharmacies in South Africa - a pilot study. Afr J Pharm Pharmacol. 2015;9(48):1099-105.

5. U.S. Food \& Drug Administration. FDA's Human Drug Compounding Progress Report: Three Years after Enactment of the Drug Quality and Security Act (January 2017). Updated June 21, 2018

6. Amanda Baltazar. Drug compounding in pharmacies: uses, benefits, and risks of uniquely formatted medication. Reviewed March 26, 2020

7. U.S. Food \& Drug Administration. Bulk drug substances used in compounding. Updated October 09, 2020

8. Medisca. What is compounding? https://www.medisca.com.au/compo unding/what-is-compounding. Accessed July, 2021

9. Cantrell SA. Improving the quality of compounded sterile drug products: A historical perspective. Ther Innov Regul Sci. 2016;50(3):266-9. https:// doi.org/10.1177/2168479015620833 (PMID: 30227062).

10. Watson CJ, Whitledge JD, Siani AM, Burns MM. Pharmaceutical compounding: a history regulatory overview, and systematic review of compounding errors. J Med Toxicol. 2021;17:197-217.

11. What is Compounding? https://dash.harvard.edu/bitstream/handle/1/ $8852177 /$ Riley.html?sequence=1. Accessed July, 2021

12. Gudeman J, Jozwiakowski M, Chollet J, Randell M. Potential risks of pharmacy compounding. Drugs R D. 2013;13(1):1-8. https://doi.org/10.1007/ s40268-013-0005-9.PMID:23526368:PMCID:PMC3627035.
13. Ureh Dibani Okoroafor. Compounding practices: before and now. Updated December 14, 2020. Accessed July, 2021

14. Feldschuh M. Compounding in community pharmacy. Aust Prescr. 2008;31(2):30-1.

15. U.S. Food \& Drug Administration. Compounding risk alerts. Updated June 07, 2019.

16. Randell MD, Duffy PJ. Risk and Liabilities of Prescribing Compounded Medications. Postgrad Med. 2014;126(4):178-80. https://doi.org/10.3810/ pgm.2014.07.2816.

17. Mullarkey T. Pharmacy compounding of high-risk level products and patient safety. Am J Health Syst Pharm. 2009;66(17 Suppl 5):S4-13. https:// doi.org/10.2146/ajhp0108b PMID: 19710449.

18. Isles M. Patient safety issues associated with the use of compounded medicines as alternatives to approved pharmaceutical products in Europe and how best practice can improve outcomes. Int J Risk Saf Med. 2020;31(3):133-44. https://doi.org/10.3233/JRS-200002 (PMID: 32538874).

19. U.S. Food \& Drug Administration. Compounding Quality Center of Excellence. Updated 12/18/2020

20. U.S. Food \& Drug Administration. Compounding Laws and Policies. Updated July 23, 2018

21. U.S. Food \& Drug Administration. Compounding Risk Alerts.Updated June $07,2019$.

22. U.S. Food \& Drug Administration. FDA alerts health care professionals and compounders of potential risks associated with the compounding of remdesivir drug products. Updated 02/04/2021

23. USP. Breeding a superbug: how poor-quality medicines fuel AMR. https:// qualitymatters.usp.org/. Update on November 13, 2020.

24. Mittal AK, Bhardwaj R, Mishra P, Rajput SK. Antimicrobials Misuse/Overuse Adverse Effect, Mechanism, Challenges and Strategies to Combat Resistance. The Open Biotechnology Journal. 2020;14:107-12.

25. Buurma H, de Smet PA, van den Hoff OP, et al. Frequency, nature and determinants of pharmacy compounded medicines in Dutch community pharmacies. Pharm World Sci. 2003;25:280-7.

26. Llor C, Bjerrum L. Antimicrobial resistance: risk associated with antibiotic overuse and initiatives to reduce the problem. Ther Adv Drug Saf. 2014;5(6):229-41. https://doi.org/10.1177/2042098614554919.

27. AlKhatib HS, Jalouqa S, Maraqa N, Ratka A, Elayeh E, AI MS. Prevalence, determinants, and characteristics of extemporaneous compounding in Jordanian pharmacies. BMC Health Serv Res. 2019;19(1):816. https://doi. org/10.1186/s12913-019-4684-y.

28. American Society of Hospital Pharmacists. ASHP technical assistance bulletin on compounding nonsterile products in pharmacies. Am J HospPharm. 1994;51:1441-8.

29. Zaid AN, Al-Ramahi R, Shahed Q, Saleh B, Elaraj J. Determinants and frequency of pharmaceutical compounding in pharmacy practice in Palestine. Int J Pharm Pract. 2012;20(1):9-14.

30. McPherson TB, Fontane PE, Jackson KD, Martin KS, Berry T, Chereson R, Bilger R. Prevalence of compounding in independent community pharmacy practice. J Am Pharm Assoc. 2006:46(5):568-73.

31. World Health Organization (WHO). Conference of Experts on the Rational Use of Drugs (1985: Nairobi, Kenya). The rational use of drugs: issues suggested for consideration. Action programme on essential drugs and vaccines. Geneva: WHO, 1985

32. World Medical Association. WMA declaration of Helsinki: ethical principles for medical research involving human subjects. JAMA. 2013;310:2191-4. https://doi.org/10.1001/jama.2013.281053.

33. Pharmacy development services. 5 benefits of pharmacy compounding. https://www.pharmacyowners.com/5-benefits-of-pharmacy-compounding. Accessed July, 2021

\section{Publisher's Note}

Springer Nature remains neutral with regard to jurisdictional claims in published maps and institutional affiliations. 